\section{P6.040 HEALTH SYSTEM RELATED FACTORS ASSOCIATED WITH ADHERENCE TO ANTIRETROVIRAL THERAPY AMONGST ADULTS LIVING WITH HIV/AIDS IN DEVELOPING COUNTRIES: A SYSTEMATIC REVIEW}

doi:10.1136/sextrans-2013-051184.1194

${ }^{1}$ R P Gajurel, ${ }^{2} \mathrm{~A}$ Poobalan, ${ }^{2} \mathrm{~B}$ Avan. 'NHS Grampion, Aberdeen, UK; ${ }^{2}$ University of Aberdeen, Aberdeen, UK

Background Although ART is one of the most celebrated treatment advances in the recent medical history, a large proportion of eligible people were not receiving ART and one fifth of patients who started ART were no longer in care/treatment after one year due to various reasons in low and middle-income countries. The objective of this study is to systematically review to identify the health system related factors associated to ART treatment adherence among adults living with HIV/AIDS in developing countries.

Methods Reviewer systematically searched MEDLINE and EMBASE databases (1996 to August 2011) and web-based information. The reference lists of included papers were also checked, with citation searching on key papers. The studies examining health system factors associated with ART treatment adherence were considered for inclusion. Quality assessment and data extraction were performed.

Results A total of 622 articles were identified, and 14 studies met the inclusion criteria, representing in 9 qualitative, 3 quantitative and 2 mixed methods and 11 countries out of 152 developing countries. Twenty-four health system themes were found associated to ART non adherence and 18 themes were found as facilitator for adherence.

Author's conclusion: The identified health system factors, service providers including clinical team can use this information to engage in open discussion with patients and PLWHAs to promote ART treatment adherence and identify issues within their own health centres. Indeed, HIV patients from developing are found very sensitive in utilisation of health services not only on the amount/ numbers of services they received, but also on time, cost, place, information and quality. Thus the responsibility of adherence shifts to the health service provider leaving patients with different choices and options.

\section{P6.041 COST ANALYSIS OF RECRUITMENT STRATEGIES USED IN THE PARTNERS PRE-EXPOSURE PROPHYLAXIS (PREP) CLINICAL TRIAL AT KISUMU SITE, KENYA}

doi:10.1136/sextrans-2013-051184.1195

A 0 Obiero, J B Odoyo, R 0 Ondondo, B K Rono, J 0 Odondi, E A Bukusi. Kenya Medical Research Institute, Nairobi, Kenya

Background Recruitment of participants into large clinical trials may be challenging and costly. Utilization of limited resources to maximise the yield is crucial. We sought to compare costs of different recruitment strategies.

Methods To recruit 600-800 HIV discordant couples, we explored different recruitment strategies: referrals from voluntary HIV counselling and testing (VCT) centres and Home based counselling and testing (HBCT) and referrals from participants involved in a previous clinical trial. In the three strategies, we implemented accelerated mobilisation through public address system, radio talk shows and the couples ambassador model (where influential peers encourage couples HIV counselling and testing). Direct cost computation was the total amount of money spent per strategy. VCT and HBCT costs included counsellors training, cost of communication and transport reimbursement for participants' visits. The previous study participants' recruitment costs included communication and transport.

Results Between August 2008 and October 2010, 2060 HIV discordant couples were referred for screening. VCT, 1666(80.9\%) from
VCT; HBCT, 345(16.7\%); and 49(2.4\%) by participants from earlier trial. The cost per strategy for VCT was $\$ 81,973$, HBCT $\$ 2,300$ and Previous study participants recruitment $\$ 327$; the yield per strategy was VCT 440/1666(26.4\%), HBCT 161/345(46.7\%) and previous study participant recruitment $28 / 49$ (57.1\%). However, VCT and HBCT contributed the most to enrollment at the study site $440 / 629(70 \%)$ and $161 / 629(26 \%)$ respectively. The average cost per couple referred from VCT was $\$ 49$, HBCT was $\$ 7$ and previous study participant recruitment was $\$ 7$. This translates to average cost of couples enrolled to $\$ 63$.

Conclusion VCT strategy was most costly compared to those from previous clinical trial and HBCT; however, it yielded the highest enrolment. Multiple strategies are recommended for successful recruitment. This computation does not take into account staff time for each of the strategies listed.

\section{P6.042 ART UPTAKE AND CD4 RECOVERY RATES POST ART INITIATION IN A COHORT OF HIV-1 INFECTED INDIVIDUALS REFERRED FOR ART AT JINJA PARTNERS PREP SITE}

doi:10.1136/sextrans-2013-051184.1196

'S Mubezi, 'S Khasaka, 'F Nsanga, 'W Musubika, 'P Ssenkungu, 1,2E Katabira, 1.3P Ndase, 'P Shuha. 'Jinja Partners PrEP Study, Makerere University, Infectious Diseases Institute, Jinja, Uganda; ${ }^{2}$ Department of Medicine, Makerere University College of Health Sciences, Kampala, Uganda; ${ }^{3}$ Department of Global Health, University of Washington, Seattle, WA, United States

Background The current WHO recommendation for ART initiation at CD4 cell count $\leq 350$ is premised on the need for achieving better immune recovery. However, not every HIV infected person meeting this threshold is able to start ART in a timely manner.

Methods We retrospectively analysed ART initiation trends and CD4 responses of HIV-1 infected participants enrolled from the Jinja, Uganda site of the Partners PrEP Study. The Partners PrEP study was a phase III, randomised, placebo-controlled trial of daily oral tenofovir and emtricitabine/tenofovir PrEP among HIV-uninfected members of HIV-1 serodiscordant relationships.

Results Between June 2009 and September 2012, 37.7\% (116/308) of HIV-1 infected participants met national threshold for ART initiation and were referred for ART initiation. Over this 39 month period, $64.7 \%(75 / 116)$ of referred participants initiated ART while $35.3 \%$ (41/116) never started ART for various reasons. 84\% (63/75) of those initiated on ART had at least two consecutive 6 monthly CD4 test results available. $79.4 \%(50 / 63)$ were on zidovudine based regimens while $20.6 \%(13 / 63)$ were on tenofovir based regimens. Median pre-ART CD4 baseline was 231 Cells/ul. After 6 months of ART initiation, $54 \%(34 / 63)$ of participants noted $>50 \%$ increase in CD4 while $33.3 \%(21 / 63)$ noted $<50 \%$ increase in CD4. After 12 months of ART initiation, $66.7 \%(42 / 63)$ of participants registered $>50 \%$ increase in CD4 while $27 \%(17 / 63)$ registered $<50 \%$ increase in CD4. $12.7 \%(8 / 63)$ and $6 \%(4 / 63)$ of participants initiated on ART showed a decline from pre-ART CD4 baseline at 6 and 12 months respectively. Tenofovir \& zidovudine based regimens were equivalent in achieving CD4 recovery.

Conclusion Our findings highlight the need for addressing potential structural and individual barriers to ART care (only $64.7 \%$ of cases referred for ART initiated treatment). Two-thirds of those initiated on ART more than doubled their CD4 counts at 12 months post-ART initiation.

\section{P6.043 SCALING UP CERVICAL CANCER SCREENING IN HIV/AIDS} RESOURCE LIMITED SETTINGS: TASO JINJA EXPERIENCE

doi:10.1136/sextrans-2013-051184.1197

C Coutinho, S Nampiija, E Musoke Seruma, S Nambafu, D Mpiima. The AIDS Support Organization(TASO) Uganda Limited, Jinja, Uganda 
Background Globally 50,000 women are diagnosed with cervical cancer,many lose their lives per year, majority of these live in resource limited countries like Uganda,the incidence rate is very high. $80 \%$ of cervical cancer cases are diagnosed in late stages. Few women screen in developing countries compared to developed countries. Cervical cancer is considered an AIDS defining illness. HIV positive women with CD4 less than 200 are at high risk of getting cervical cancer.

\section{Program description}

It's under this back ground that Mild May funded by CDC trained health workers to screen for cervical cancer using the affordability method of visual inspection with acetic acid, treat and manage positive lesions using CRYOTHERAPY. Logistics were delivered and work started on 22rd August2012 after community mobilisation and referrals. By December 2012 a total of 214 clients where screened of which $47.7 \%$ were HIV positive and $52 \%$ were HIV negative. $19.2 \%$ of clients screened for Cancer of the cervix had positive lesions of which $53.7 \%$ were HIV positive and $46.3 \%$ were HIV negative; $79.9 \%$ had negative results of which $48.5 \%$ were HIV positive and $52.6 \%$ were HIV negative; $0.9 \%$ had suspicious lesions.

Lessons learnt

It is important to integrate cervical cancer screening within HIV/AIDS Care setting alongside family planning. It is an entry point for diagnosis and treatment of STI. More gynaecological conditions have been identified, managed and some referred to gynaecologist for specialised management.

It strengthens partnership with stake holders through support and supervision, collaboration and networking.

Stigma to women who are HIV negative or whose serological status is unknown shun away from screening in an HIV/AIDS Care setting.

Conclusion The best way to prevent cervical cancer is by early screening and treatment of precancerous lesions; early diagnosis and treatment of cervical cancer thus reducing mortality rate among women.

\section{P6.044 SCALING UP STI RESOURCE AND EDUCATIONAL CAPACITY FOR GENERAL PRACTISE IN NEW SOUTH WALES, AUSTRALIA}

doi:10.1136/sextrans-2013-051184.1198

1,2C Bourne, 'C Murray. 'NSW STI Programs Unit, Sydney, Australia; ${ }^{2}$ School of Public Health and Community Medicine, University of NSW, Sydney, Australia

Background Increasing access to STI resources and education for general practise (GP) and improving referral pathways to specialised STI care were key objectives of the 2006 STI Strategy, New South Wales (NSW). Prior GP STI education and support was ad hoc without strategic direction. No identifiable STI support or training was provided to GP nurses. In 2008 chlamydia was the most commonly reported STI, 200 per $10^{5}$ and annual GP chlamydia testing rate for $<30$ yo, $7 \%$.

Methods A multidisciplinary GP working group was created within the the newly established NSW STI Programs Unit in 2007. The group coordinated needs identification and development of resources and online and participatory learning packages for NSW GP. Recognised private and non-government GP training providers were identified to host and assist with training coordination for doctors and nurses.

Results STI management 'tools' and training modules focussed on chlamydia, the most common STI in NSW. Simple chlamydia testing and treatment provided an easy introduction to STI care. Resources developed include brief and comprehensive online training modules; locally facilitated, in person learning modules; tools for practise audit, STI testing in priority populations, partner notification and referral pathways; NSW Sexual Health Information telephone Line; competency standards for GP nurses. Resource and training promotion occurred through professional discipline newsletters and journal editorials, interviews and papers; 'master classes' and booths at conferences; and directly to individual doctors, including the NSW Chief Health Officer communique reminding GP of their responsibilities with STI partner notification. An external academic process and impact evaluation of the project revealed important GP learning preferences. Chlamydia testing rates have yet to be reassessed. Project scale awaits the results of a cluster randomised control trial, ACCEPt http://www.accept.org.au/

Conclusion A strategic approach to GP resource and training capacity development has been strengthened by concurrent planning, implementation and programme management.

\section{P6.045 WITHDRAWN BY AUTHOR}

\section{P6.046 INTEGRATION OF STI DIAGNOSTICS AND TREATMENT PROGRAMIMES AND HIV PREVENTION PROGRAMMES FOR VULNERABLE GROUPS}

doi:10.1136/sextrans-2013-051184.1199

0 Savenko, S Filippovych, Z Islam. International HIVIAIDS in Ukraine, Kiev, Ukraine

Problem: Ukraine has the fastest HIV/STI spread rate in Europe (221 806 PLWH as of November 2012).

Sexual HIV transmission mode has been dominant in Ukraine since 2008 (51\% - sexual mode, $28 \%$-parenteral).

HIV/STI epidemics in Ukraine are concentrated within vulnerable groups and threaten to generalise.

Activities description:

STI diagnostics and treatment programmes for vulnerable groups started in Ukraine since 2008, when the situation was unfavourable due to lack of understanding between medical services, adverse attitude towards case management and integrated services principles. Several models of dermatovenerological assistance to the vulnerable groups and their stage-by-stage implementation were developed.

Results In $200862 \mathrm{HCFs}$ and 82 NGOs joined the programme.

In 2012 STI diagnostics and treatment became available in 108 healthcare facilities of Ukraine (50 dermatovenerological dispensaries, 25 AIDS centres, 33 general clinics).

As of 31.07.2012 there were 595812 screening tests and counselling for vulnerable groups on STI and viral hepatitis and 33637 STI treatment courses were provided.

193247 vulnerable groups' representatives (as of 31.07.2012) were referred to 15 mobile clinics providing HIV/STI testing and counselling for vulnerable groups.

16 trainings were held for NGOs and healthcare facilities' representatives.

In 201222 multidisciplinary teams were created providing STI diagnostics and treatment for vulnerable groups in healthcare facilities.

\section{Conclusions}

1. STI diagnostics and treatment services should be an integral part of the integrated HIV prevention services package for vulnerable groups.

2. STI programmes can be implemented only in cooperation with the dermatovenerological service and the AIDS service.

3. Programs should be implemented simultaneously under several models considering country and regional peculiarities.

4. MDTs are the most successful model.

P6.047 ISSUES AND BARRIERS TO HEALTHCARE SERVICE ACCESS
AMONG MEN WHO HAVE SEX WITH MEN IN INDIA: A QUALITATIVE PROSPECTIVE

doi:10.1136/sextrans-2013-051184.1200 